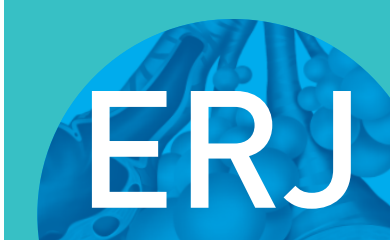

open research
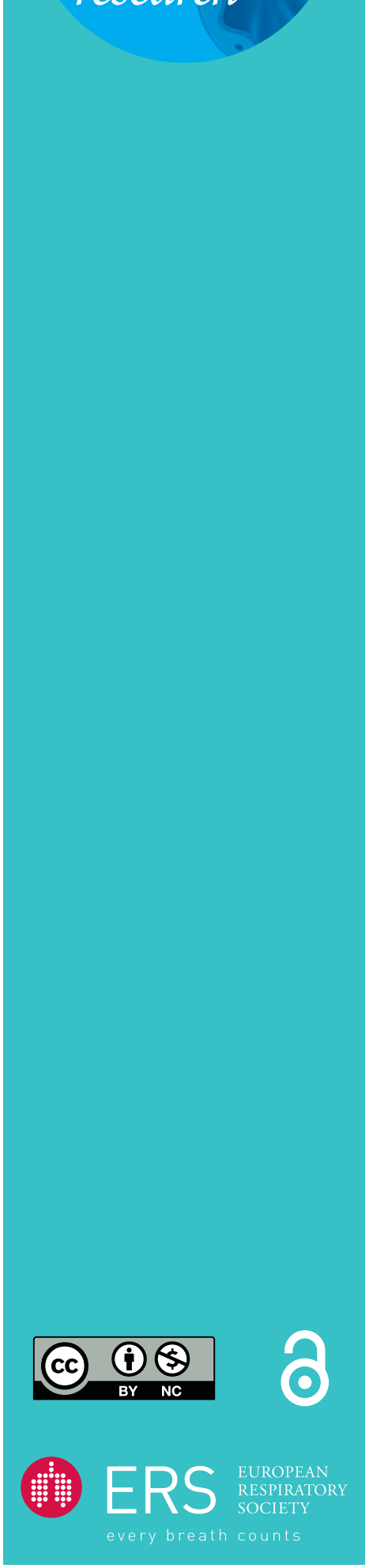

\section{Pulmonary veno-occlusive disease is highly prevalent in scleroderma patients undergoing lung transplantation}

\author{
To the Editor:
}

Pulmonary complications including pulmonary hypertension (PH) and interstitial lung disease (ILD) are leading causes of mortality in systemic sclerosis/scleroderma (SSc) [1]. PH associated with SSc (SSc-PH) has been attributed to World Health Organization (WHO) group 1 disease, pulmonary arterial hypertension (SSc-PAH); WHO group 2 due to left ventricular systolic or diastolic dysfunction (SSc-PVH); or WHO group 3 related to interstitial lung disease (SSc-PH-ILD) [2, 3]. SSc-PH is found in $10-12 \%$ of SSc patients [4]. Of the 71 out of 237 patients in the PHAROS (Pulmonary Hypertension Assessment and Recognition of Outcomes in Scleroderma) cohort who had PH, 69\% had SSc-PAH, 10\% had SSc-PVH and 21\% had SSc-PH-ILD [1]. SSc-PAH has poor prognosis and less favourable response to pulmonary vasodilator therapy compared to idiopathic pulmonary arterial hypertension (IPAH) [5, 6]. SSc-PH-ILD is a strong predictor of death compared to SSc-PAH or ILD alone [7].

Pulmonary veno-occlusive disease (PVOD) is a rare cause of group $1 \mathrm{PH}$ (WHO Group 1'), and is difficult to distinguish clinically from IPAH $[8,9]$. The histological hallmark is obstructive intimal fibrosis of small veins and venules compared to the arteriolar pathology of $\mathrm{PAH}$, and prognosis is extremely poor [10].

Studies have shown that SSc patients with PAH have a worse prognosis and response to PAH therapies, and reports have shown an association between PVOD and SSc-PAH [11-14]. However, the prevalence of PVOD in SSc-PH-ILD is heretofore unknown. We hypothesised that SSc-PH seen in both SSc-PAH and SSc-PH-ILD may be associated with PVOD-like pathology, which could explain the worse prognosis in this population.

A retrospective study of SSc patients with diagnosis of $\mathrm{PH}$ was performed on sequential patients transplanted between January 1, 2007, and October 1, 2013. Exclusion criteria included misdiagnosis of SSc, absence of $\mathrm{PH}$ or missing pathological slides. $\mathrm{PH}$ was defined as mean pulmonary artery pressure $\geqslant 25 \mathrm{mmHg}$ on right heart catheterisation. Control samples were obtained from transplanted patients without SSc who had IPAH $(n=3)$ or PVOD $(n=3)$.

For histopathological analysis, haematoxylin and eosin and Verhoeff-Van Gieson stains were prepared from one block per lobe (thus, two blocks for single lung transplant (one each from the upper and lower lobes) and four blocks for double lung transplant (one from the upper and lower lobes of each lung). PVOD pattern was classified as absent $(0)$, mild $(1+)$ or moderate-severe $(2+)$. Pulmonary veins and venules were examined for changes of PVOD: intimal thickening, obstructive fibrous luminal septa or recanalisation and arterialisation of pulmonary veins. Angioma-like lesions were reported in capillaries. Pulmonary arteries were evaluated for plexiform lesions, eccentric intimal fibrosis, concentric intimal fibrosis, recanalising thrombosis and muscularisation of small arteries. In addition to vascular pathology, the pulmonary interstitium was examined for usual interstitial pneumonia, nonspecific interstitial pneumonia, emphysema and pneumonitis. Comprehensive vessel morphometry was also performed on

@ERSpublications

There is an unexpectedly high incidence of PVOD in patients with SSc-PH-ILD. Presence of PVOD may be an unrecognised contributor to the dismal prognosis of these patients. Early transplant referral should be considered for those with SSc-PH-ILD. http://ow.ly/vPvc30neJZV

Cite this article as: Gupta S, Gupta A, Rehman S, et al. Pulmonary veno-occlusive disease is highly prevalent in scleroderma patients undergoing lung transplantation. ERJ Open Res 2019; 5: 001682018 [https://doi.org/10.1183/23120541.00168-2018].

Copyright @ERS 2019. This article is open access and distributed under the terms of the Creative Commons Attribution Non-Commercial Licence 4.0. 
a)
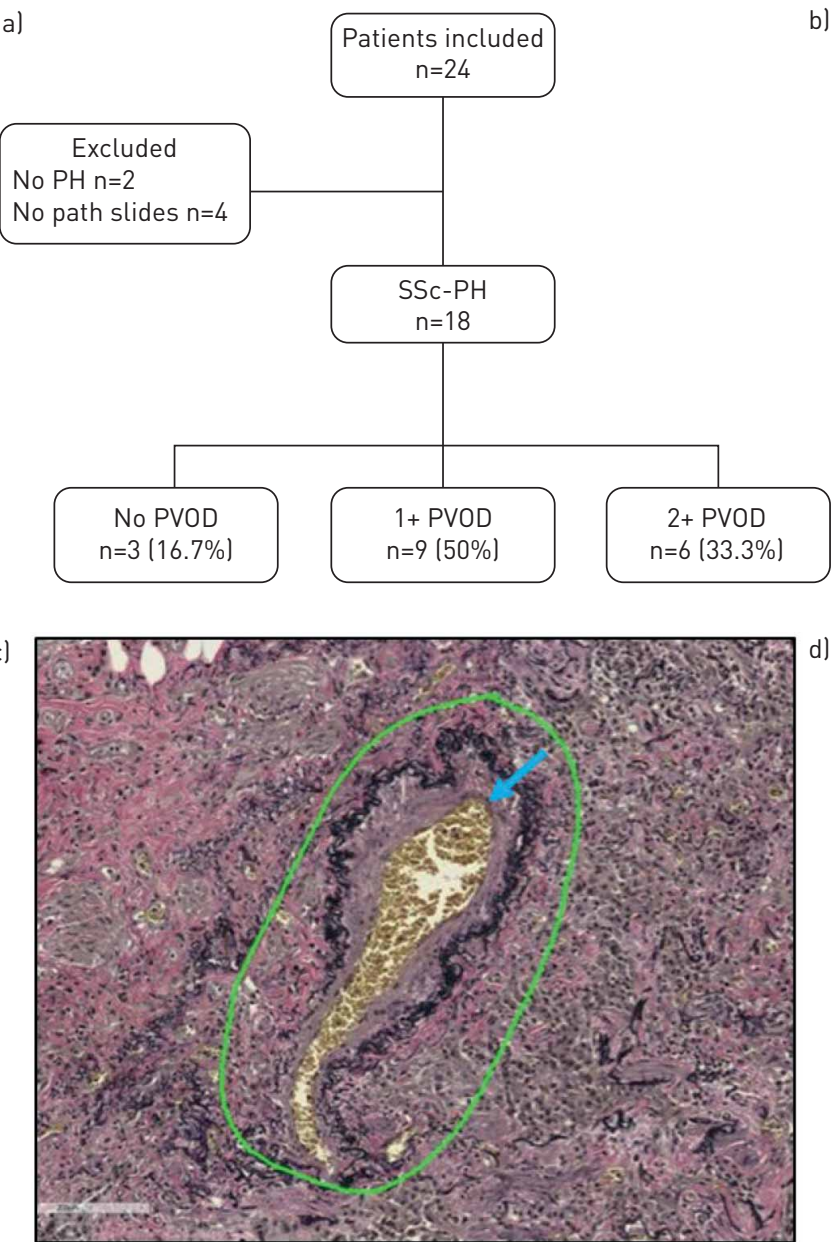

b)

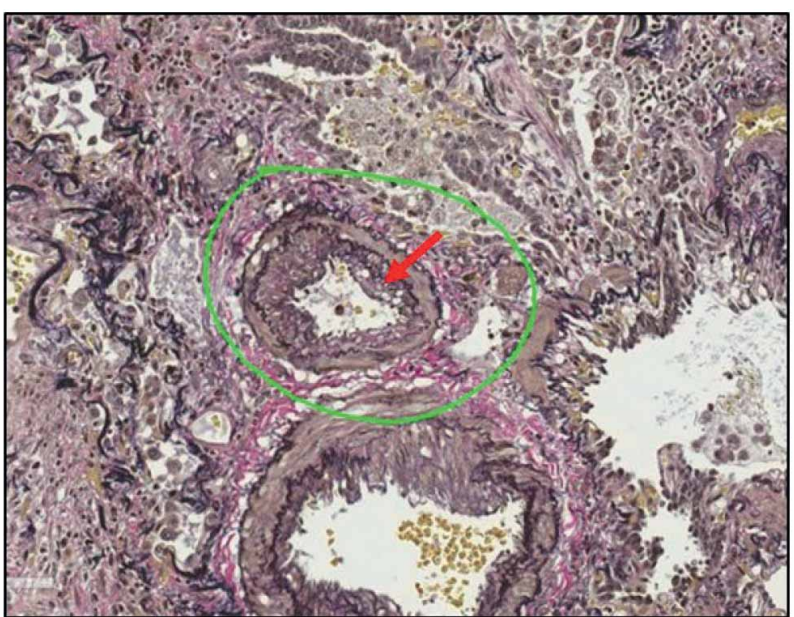

d)

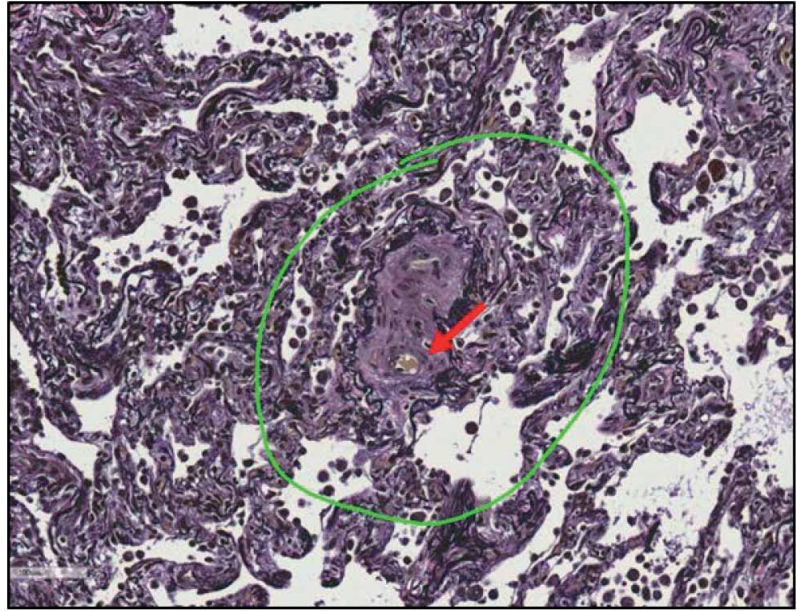

FIGURE 1 a) Study profile: of 24 scleroderma (SSc) patients studied, 18 were enrolled. Of those, nine had 1+ pulmonary veno-occlusive disease (PVOD) and six had 2+ PVOD, while PVOD was absent in the remaining three. b) Pulmonary arteriole in a patient with SSc-PVOD showing changes of intimal fibrosis (red arrow). c and d) Pulmonary vein in a patient with SSc-PVOD depicting a partially arterialised vein (blue arrow) and intimal fibrosis (red arrow) consistent with changes of PVOD. PH: pulmonary hypertension.

five arterioles and venules per lobe of the lung. Measurements included external and lumen diameter, and smooth muscle and intimal thickness.

Institutional review board approval was obtained from University of Pittsburgh (Pittsburgh, PA, USA) for completion of the study.

The continuous variables were analysed with Kwallis and categorical variables with Fisher's exact test. A p-value $<0.05$ was considered statistically significant. All analyses were performed with STATA (StataCorp, College Station, TX, USA).

Of 24 SSc patients who received tranplants, 18 SSc patients met the inclusion criteria for the study and they all had SSc-PH-ILD. Of these, nine had 1+ PVOD and six had 2+ PVOD. There was no significant difference in demographics amongst the groups, though the odds of having one higher category of PVOD increased 10-fold in those with limited versus diffuse scleroderma (95\% CI $0.77-133.4, \mathrm{p}=0.07$ ) (figure 1 and table 1). Echocardiography was reviewed in all patients and there were no differences in right ventricular systolic pressure (table 1). Diffusing capacity was significantly lower in patients with PVOD (table 1). There was no significant difference in other pulmonary function test parameters, 6-min walk test distance and need for oxygen.

Patients with PVOD had lower pulmonary artery pressures compared to those with no PVOD (table 1). There was no significant difference in cardiac output/index or pulmonary vascular resistance among the groups. One of the 1+ PVOD patient had pulmonary arterial wedge pressure $17 \mathrm{mmHg}$ and pulmonary vascular resistance 1.5 Wood units. 


\begin{tabular}{|c|c|c|c|c|c|c|}
\hline \multicolumn{7}{|l|}{ TABLE 1} \\
\hline Age years & $50.3 \pm 14$ & $52.2 \pm 9.5$ & $54 \pm 8.4$ & $42.6 \pm 15$ & $38.3 \pm 14$ & 0.38 \\
\hline Females & $1(33.3 \%)$ & $4(44.4 \%)$ & $2(33.3 \%)$ & $2(66.7 \%)$ & $2(66.7 \%)$ & 0.9 \\
\hline Caucasians & $1(33.3 \%)$ & $8(88.9 \%)$ & $5(83.3 \%)$ & $3(100 \%)$ & $3(100 \%)$ & 0.2 \\
\hline Smoking history pack-years & $2.3 \pm 2$ & $13 \pm 20$ & $3.3 \pm 8$ & 0 & 0 & 0.3 \\
\hline Diffuse SSc & 2 & 3 & 1 & & & 0.2 \\
\hline Limited SSc & 0 & 3 & 4 & & & \\
\hline RVSP mmHg & $68 \pm 20$ & $43.8 \pm 24$ & $75.4 \pm 32$ & $83 \pm 12$ & $61.3 \pm 32$ & 0.18 \\
\hline FVC $\%$ predicted & $45.6 \pm 21$ & $51.3 \pm 20$ & $48.3 \pm 20$ & $77.9 \pm 17$ & $63.5 \pm 20$ & 0.2 \\
\hline TLC & $24 \pm 13$ & $27.3 \pm 7$ & $26.6 \pm 18$ & $73.6 \pm 14$ & $47.2 \pm 28$ & 0.1 \\
\hline PAWP mmHg & $9 \pm 5.5$ & $10.6 \pm 3.5$ & $9.6 \pm 3.1$ & $12 \pm 2.6$ & $4.7 \pm 2$ & 0.1 \\
\hline $\mathrm{TPG} \mathrm{mmHg}$ & $34.6 \pm 0.5$ & $21.5 \pm 12$ & $26.3 \pm 11$ & $45 \pm 8$ & $50.6 \pm 12$ & 0.01 \\
\hline PVR WU & $4.9 \pm 0.9$ & $4.9 \pm 3.3$ & $4.5 \pm 1.7$ & $14.9 \pm 8.7$ & $10.1 \pm 4$ & 0.07 \\
\hline \multicolumn{7}{|l|}{ Pathology } \\
\hline \multicolumn{7}{|l|}{ Pulmonary veins } \\
\hline Intimal thickening & 0 & $1(11.1 \%)$ & $5(100 \%)$ & 0 & $3(100 \%)$ & $<0.001$ \\
\hline Obstructive fibrous luminal septa or recanalisation & 0 & $4(44.4 \%)$ & $5(100 \%)$ & $1(33.3 \%)$ & $3(100 \%)$ & 0.002 \\
\hline Arterialisation of pulmonary veins & 0 & $7(77.8 \%)$ & $5(100 \%)$ & $1(33.3 \%)$ & $3(100 \%)$ & 0.02 \\
\hline \multicolumn{7}{|l|}{ Capillaries } \\
\hline Angioma-like lesions in capillaries & 0 & 0 & $1(20 \%)$ & $1(33.3 \%)$ & $3(100 \%)$ & 0.006 \\
\hline \multicolumn{7}{|l|}{ Pulmonary arteries } \\
\hline Plexiform lesions & 0 & 0 & 0 & $3(100 \%)$ & 0 & 0.002 \\
\hline \multicolumn{7}{|l|}{ Pulmonary arteries } \\
\hline Smooth muscle \% & $25.4 \pm 2.5$ & $29.7 \pm 4$ & $31.7 \pm 7.5$ & $36.0 \pm 10$ & $26.0 \pm 4.4$ & 0.2 \\
\hline Intima \% & $36.4 \pm 0.2$ & $32.0 \pm 8.7$ & $45.7 \pm 16.5$ & $30.5 \pm 8$ & $30.4 \pm 19.4$ & 0.4 \\
\hline Ratio of intima to smooth muscle & $1.6 \pm 0.09$ & $2.0 \pm 2.7$ & $1.5 \pm 0.2$ & $1.0 \pm 0.3$ & $1.2 \pm 0.8$ & 0.4 \\
\hline \multicolumn{7}{|c|}{$\begin{array}{l}\text { Comparison of the demographics, haemodynamics and pathology of scleroderma (SSc)-pulmonary hypertension (PH)-interstitial lung disease } \\
\text { patients. Idiopathic pulmonary arterial hypertension (IPAH) and pulmonary veno-occlusive disease (PVOD) were used as controls. Data are } \\
\text { presented as mean } \pm \text { SD, unless otherwise stated. p-values were obtained by comparing all cases and controls using Kruskal-Wallis for } \\
\text { continuous variables and Fisher's exact test for categorical variables. Bold indicates statistically significant p-values. NYHA: New York Heart } \\
\text { Association; RVSP: right ventricular systolic pressure; FVC: forced vital capacity; TLC: total lung capacity; DLCO: diffusing capacity of the lung } \\
\text { for carbon monoxide; PA: pulmonary artery; PAWP: pulmonary arterial wedge pressure; TPG: transpulmonary pressure gradient; PVR: } \\
\text { pulmonary vascular resistance; WU: Wood units. }\end{array}$} \\
\hline
\end{tabular}

Compared to IPAH, no plexiform lesions were seen in SSc patients (table 1). The pulmonary vein smooth muscle percentage was lower in 2+ PVOD. See table 1 for details in differences in the pathology characteristics and morphometric analysis between different groups.

Our study suggests that there is a high incidence of PVOD in patients with SSc-PH-ILD. Also, the prevalence is higher in those with limited versus diffuse SSc. This finding of PVOD may be an unrecognised contributor to the dismal prognosis of these patients.

Interestingly, plexiform arteriopathy, a hallmark of IPAH, was not seen in any of the SSc-PH-ILD patients. Recent studies have reported an association of PVOD with SSc-PAH [11, 12]. Overbeek et al. [13] described eight patients with SSc-PAH, of whom four had changes consistent with PVOD. None of these 
had plexiform changes compared to 10 IPAH patients analysed who all had plexiform lesions. DoRFMULLER et al. [14] studied eight patients with connective tissue disease-induced PAH, of whom four had limited SSc. Six of the eight patients had PVOD changes. Based on our literature search, all studies reported patients with SSc-PAH and there were no studies that specifically looked at SSc-PH-ILD.

SSc-PH-ILD has five-fold increased risk of death compared to SSc-PAH. LE PAVEC et al. [7] reported no clear benefit of PAH therapy in SSc-PH-ILD patients and extremely poor survival. The poor prognosis in this population has been attributed to worsening ventilation-perfusion mismatch. Our results suggest that a higher prevalence of PVOD may also play a role. Early transplant referral should be considered in these patients as they are refractory to pulmonary vasodilator therapy.

The higher than expected incidence of PVOD in SSc-PH-ILD patients could be due to selection bias in using explanted lungs, as these patients might have deteriorated faster than those without PVOD and may have been refractory to pulmonary vasodilator therapy, thus requiring lung transplantation. Eukaryotic translational initiation factor $2 \alpha$ kinase $(E I F 2 A K 4)$ gene and bone morphogenetic protein receptor type II (BMPR2) gene mutations have been reported with PVOD. We do not have information to investigate the genetic basis of this in our patient population. Further studies are needed.

In conclusion, our study is the first to highlight the high prevalence of PVOD in patients with SSc-PH-ILD, potentially contributing to the poor prognosis of this disease.

\section{Shikha Gupta ${ }^{1}$, Aman Gupta ${ }^{2}$, Sofiya Rehman ${ }^{3}$, Iclal Ocak ${ }^{4}$, Robyn T. Domsic ${ }^{5}$, Frank Schneider ${ }^{6}$ and M. Patricia George ${ }^{7}$}

${ }^{1}$ Division of Pulmonary, Allergy and Critical Care Medicine, Dept of Medicine, UPMC, Pittsburgh, PA, USA. ${ }^{2}$ Dept of General Internal Medicine, UPMC, Pittsburgh, PA, USA. ${ }^{3}$ Dept of Internal Medicine, UPMC, Mckeesport, PA, USA. ${ }^{4}$ Thoracic Imaging Division, Dept of Radiology, UPMC, Pittsburgh, PA, USA. ${ }^{5}$ Division of Rheumatology and Clinical Immunology, Dept of Medicine, UPMC, Pittsburgh, PA, USA. ${ }^{6}$ Dept of Pathology, UPMC, Pittsburgh, PA, USA. ${ }^{7}$ Division of Pulmonary Medicine, Dept of Medicine, National Jewish Health, Denver, CO, USA.

Correspondence: Shikha Gupta, 1671 Biltmore Ln, Pittsburgh, PA 15217, USA.

E-mail: guptashikha.dr@gmail.com

Received: Oct 012018 | Accepted after revision: Dec 102018

Author contributions: S. Gupta, R.T. Domsic and M.P. George designed the study. S. Gupta, A. Gupta and S. Rehman collected the data. I. Ocak reviewed the computed tomography images. F. Schneider performed the pathological analysis. S. Gupta and A. Gupta performed the morphometric analysis. S. Gupta and A. Gupta analysed and interpreted the data, and wrote the manuscript, under the guidance of M.P. George. All authors reviewed and approved the manuscript.

Conflict of interest: S. Gupta has nothing to disclose. A. Gupta has nothing to disclose. S. Rehman has nothing to disclose. I. Ocak has nothing to disclose. R.T. Domsic has nothing to disclose. F. Schneider has nothing to disclose. M.P. George reports receiving personal fees from Actelion and Bayer for consulting on medical advisory boards and speaking about nonbranded, disease-specific topics; and from Gilead for speaking about nonbranded, disease-specific topics, all outside the submitted work.

\section{References}

1 Hinchcliff M, Fischer A, Schiopu E, et al. Pulmonary Hypertension Assessment and Recognition of Outcomes in Scleroderma (PHAROS): baseline characteristics and description of study population. J Rheumatol 2011; 38: 2172-2179.

2 Badesch DB, Champion HC, Sanchez MA, et al. Diagnosis and assessment of pulmonary arterial hypertension. J Am Coll Cardiol 2009; 54: Suppl., S55-S66.

3 Galie N, Palazzini M, Manes A. Pulmonary hypertension and pulmonary arterial hypertension: a clarification is needed. Eur Respir J 2010; 36: 986-990.

4 Chaisson NF, Hassoun PM. Systemic sclerosis-associated pulmonary arterial hypertension. Chest 2013; 144: $1346-1356$.

5 Lammi MR, Mathai SC, Saketkoo LA, et al. Association between initial oral therapy and outcomes in systemic sclerosis-related pulmonary arterial hypertension. Arthritis Rheumatol 2016; 68: 740-748.

6 Chung L, Domsic RT, Lingala B, et al. Survival and predictors of mortality in systemic sclerosis-associated pulmonary arterial hypertension: outcomes from the pulmonary hypertension assessment and recognition of outcomes in scleroderma registry. Arthritis Care Res (Hoboken) 2014; 66: 489-495.

7 Le Pavec J, Girgis RE, Lechtzin N, et al. Systemic sclerosis-related pulmonary hypertension associated with interstitial lung disease: impact of pulmonary arterial hypertension therapies. Arthritis Rheum 2011; 63: 2456-2464.

8 Simonneau G, Gatzoulis MA, Adatia I, et al. Updated clinical classification of pulmonary hypertension. J Am Coll Cardiol 2013; 62: Suppl., D34-D41. 
9 Masters K, Bennett S. Pulmonary veno-occlusive disease: an uncommon cause of pulmonary hypertension. $B M J$ Case Rep 2013; 2013: bcr2012007752.

10 Pietra GG, Capron F, Stewart S, et al. Pathologic assessment of vasculopathies in pulmonary hypertension. J Am Coll Cardiol 2004; 43: Suppl. S, 25s-32s.

11 Daraban AM, Enache R, Predescu L, et al. Pulmonary veno-occlusive disease: a rare cause of pulmonary hypertension in systemic sclerosis. Case presentation and review of the literature. Rom J Intern Med 2015; 53: 175-183.

12 Harch S, Whitford H, McLean C. Failure of medical therapy in pulmonary arterial hypertension. Is there an alternative diagnosis? Chest 2009; 135: 1462-1469.

13 Overbeek MJ, Vonk MC, Boonstra A, et al. Pulmonary arterial hypertension in limited cutaneous systemic sclerosis: a distinctive vasculopathy. Eur Respir J 2009; 34: 371-379.

14 Dorfmuller P, Humbert M, Perros F, et al. Fibrous remodeling of the pulmonary venous system in pulmonary arterial hypertension associated with connective tissue diseases. Hum Pathol 2007; 38: 893-902. 